\title{
Parental education and lung function of children in the PATY study
}

\author{
Hana Slachtova • Ulrike Gehring • Gerard Hoek • Hana Tomaskova • \\ Heike Luttmann-Gibson · Hanns Moshammer • Anna Paldy • Sam Pattenden • \\ Katarina Slotova $\cdot$ Frank Speizer $\cdot$ Renata Zlotkowska $\cdot$ Joachim Heinrich
}

Received: 28 May 2010/Accepted: 14 September 2010/Published online: 30 September 2010

(C) The Author(s) 2010. This article is published with open access at Springerlink.com

\begin{abstract}
Studies of the relationships between low socioeconomic status and impaired lung function were conducted mainly in Western European countries and North America. East-West differences remain unexplored. Associations between parental education and lung function were explored using data on 24,010 school-children from eight cross-sectional studies conducted in North America, Western and Eastern Europe. Parental education was defined as low and high using country-specific classifications. Country-specific estimates of effects of low parental education on volume and flow parameters were obtained using linear and logistic regression, controlling for early life and other individual risk factors. Meta-regressions were used for assessment of heterogeneity between country-specific estimates. The association between low parental education and
\end{abstract}

Ethics Committee Approval: The paper combines the data from the eight previous cross-sectional studies-all of them were approved by the Ethics Committees.

H. Slachtova $(\bowtie) \cdot H$. Tomaskova

Center of Health Services, Institute of Public Health,

Partyzanske nam. 7, 70200 Ostrava, Czech Republic

e-mail: hana.slachtova@zu.cz

U. Gehring · G. Hoek

Institute for Risk Assessment Sciences, Utrecht University,

Utrecht, The Netherlands

H. Luttmann-Gibson · F. Speizer

Department of Environmental Health, Harvard School of Public

Health, Boston, MA, USA

H. Moshammer

Institute of Environmental Health, Medical University of

Vienna, Vienna, Austria

A. Paldy

National Institute of Environmental Health, Budapest, Hungary lung function was not consistent across the countries, but showed a more pronounced inverse gradient in the Western countries. The most consistent decrease associated with low parental education was found for peak expiratory flow (PEF), ranging from -2.80 to $-1.14 \%$, with statistically significant associations in five out of eight countries. The mean odds ratio for low PEF ( $<75 \%$ of predicted) was 1.34 (95\% CI 1.06-1.70) after all adjustments. Although social gradients were attenuated after adjusting for known risk factors, these risk factors could not completely explain the social gradient in lung function.

Keywords Lung function - Parental education . Combined analysis - East-West differences · PATY study · Children

\author{
S. Pattenden \\ London School of Hygiene and Tropical Medicine, London, UK \\ K. Slotova \\ Regional Authority of Public Health, Banska Bystrica, Slovak \\ Republic \\ R. Zlotkowska \\ Institute of Occupational Medicine and Environmental Health, \\ Sosnowiec, Poland \\ J. Heinrich \\ Institute of Epidemiology, Helmholtz Zentrum München, \\ Neuherberg, Germany \\ H. Slachtova $\cdot$ H. Tomaskova \\ Faculty of Medicine, University of Ostrava, Ostrava, Czech \\ Republic
}




$\begin{array}{ll}\text { Abbreviations } \\ \text { A } & \text { Austria } \\ \text { BMI } & \text { Body mass index } \\ \text { CI } & \text { Confidence interval } \\ \text { CZE } & \text { Czech Republic } \\ \text { FEV1 } & \text { Forced expiratory volume in the first second } \\ \text { FVC } & \text { Forced vital capacity } \\ \text { GER } & \text { Germany } \\ \text { HUN } & \text { Hungary } \\ \text { LBW } & \text { Lower birth weight } \\ \text { LRI } & \text { Low respiratory tract infection } \\ \text { MMEF } & \text { Maximal mean expiratory flow } \\ \text { NL } & \text { the Netherlands } \\ \text { OR } & \text { Odds ratio } \\ \text { PATY } & \text { Pollution and the Young (project) } \\ \text { PEF } & \text { Peak expiratory flow } \\ \text { POL } & \text { Poland } \\ \text { SES } & \text { Socioeconomic status } \\ \text { SLO } & \text { Slovakia } \\ \text { US/USA } & \text { North America }\end{array}$

\section{Introduction}

Socio-economic status (SES) has been found an important predictor of health within developed countries. Publication of the Whitehall study results [1], reporting differences in all-cause mortality between social classes, was followed by an increased interest in the issue of social health inequalities in epidemiological research [2,3].

Only a limited number of studies have investigated the SES effect on impaired lung function or chronic obstructive pulmonary disease. Studies on adults found evidence that the level of education was associated with airways disorders independently of smoking $[4,5]$. A review by Hegewald and Crapo [6] found significantly negative correlations between lung function and SES both in children and adults.

The CARDIA study [7] on young adults found a decline of lung function over time due to lower childhood SES. A British study [8] confirmed that childhood poverty was associated with poorer lung function in women aged 60-79.

American studies with large sample size $[9,10]$ also brought evidence that low SES was associated with lower lung function in children.

An Austrian study of school age children [11] found that most of lung function measures were negatively associated with a lower birth weight (LBW), lower maternal education, smaller gestational age, and larger family size.

Associations between low SES and impaired lung function both in children and adults were detected mainly in Western European countries and North America. EastWest differences have not yet been explored.
The Pollution and the Young (PATY) project offers a unique opportunity to investigate the relationship between lung function in school-age children and their socioeconomic status in a large international dataset.

\section{Materials and methods}

Study design and study population

The present study is a combined analysis of questionnaire and lung function data from eight cross-sectional studies in which the lung function measurements were part of the original studies. The study sample contained 24,010 school-children aged 6-12 years from the North American 24-cities study [12], the East European CESAR study countries [13], and data from Austria (Linz study [14]), Germany (Bitterfeldt, Hettstedt and Zerbst study [15]), and the Netherlands (24-schools study [16]). The number of children derived from the original studies into a current analysis is slightly smaller due to missing data-only those with a full set of data were taken into the analysis. The studies were conducted across the 1990s. Some studies were conducted in several areas of one country, whereas others (CESAR and the 24-cities study) were conducted in more than one country.

The combined analyses used original data including measures of lung function, and questionnaire information on a wide range of potential risk factors including sources of indoor air pollution, SES, and family medical history.

Definition of parental education status

Parental education was defined as the maximum of the mother's and father's educational level and classified into three categories (low, medium, and high) as described previously [17]. This classification was done at country level, taking frequency distributions into account to make sure that every country had a sufficiently large number of subjects with low and high parental education, respectively [17]. Nevertheless, for the present analyses the frequency of "low lung function" (see below) was found to be too small in low parental education category (as defined by Gehring et al. [17]) in some countries and therefore, the low and medium parental education categories were joined and defined as "low". Only subjects with a defined parental education were included in the analysis.

Lung function outcomes

Lung function testing was performed according to the protocol of the American Thoracic Society [18], except for the minimum exhalation time of $6 \mathrm{~s}$ (not feasible for 
children) and except for the Dutch study, which followed the protocol of the European Respiratory Society [19].

We analyzed the following volume and flow parameters: forced vital capacity (FVC), forced expiratory volume in the first second (FEV1), peak expiratory flow (PEF) and maximal mean expiratory flow (MMEF). MMEF data were not available from Germany.

In addition, "low lung function" was analyzed, specified by low FVC (defined as less than $85 \%$ of predicted by age, gender, height, and weight from all study participants from the same country), low FEV1 (less than $85 \%$ of predicted), low PEF (less than $75 \%$ of predicted) and low MMEF (less than $75 \%$ of predicted) [20]. These cut-off levels were based on a priori clinical experience. Effect on poor lung function was analyzed by logistic regression.

Potential confounders

A set of a-priori confounders was prepared based on the literature. Confounders were divided into two groups: early life factors such as maternal smoking during pregnancy, premature birth ( $<36$ weeks), low birth weight (less than $2.3 \mathrm{~kg}$ ), breastfeeding (less/more than 3 months), attendance of kindergarten, low respiratory tract infection (LRI) in first 2 years of life, and parental history of asthma; and other potential confounders like body mass index (BMI) [21], the presence of any smoker in the household, pet ownership ever, overcrowding, presence of mould/moisture in the child's home, use of gas for cooking or gas/kerosene/ oil heater and infection prior to test.

\section{Statistical analysis}

Since lung growth during childhood is not linear, the lung function variables were log-transformed for calculation of predicted values, i.e. the linear (for continuous outcomes) regression models used the natural logarithm of each lung function variable as the dependent variable and the relationship with body parameters were described using the natural logarithm of age and weight including an interaction between sex and natural logarithm of height.

Data were analyzed in two stages. In stage one, countryspecific estimates of effects of low parental education were estimated using linear regression for the continuous outcomes and logistic regression for the binary outcomes. The models included random effects at area level allowing for geographic clustering of outcomes.

In stage two, these country specific effect-estimates and their standard errors were pooled using meta-analytic methods, obtaining a mean estimate and a measure and Cochran $\chi^{2}$ test of heterogeneity. Country-specific effects are assumed to follow a random distribution about a mean, and the estimation of this mean (and confidence interval) takes into account variations both within and between studies [22].

All country-specific and pooled estimates were produced in three models-Model 1 controlled for age, sex, weight, height, and technician (technician who performed the lung function testing). In Model 2 early life factors were added; and in the third model the other potential confounders were considered (Table 1).

The magnitude of the effect of each confounder was tested separately. The final set of confounders was selected for all potential confounders that change odds ratios (OR) for any of the low lung function indicators by more than at least $10 \%$ in one country (Table 1 ).

As indicated, not all potential confounding variables were available for all studies-sensitivity analyses were conducted to assess the impact of unavailability of covariates, by comparing results between studies with full set and restricted sets of confounders. As the US data represents the largest group further sensitivity analysis was performed on the restricted data set without American data.

Meta-regressions were used to assess whether the following study characteristics could explain heterogeneity between country-specific estimates: East-West differences based upon a hypothesis of different health status in Western countries and former Eastern block (Germany was coded Eastern country as the study was conducted in the Eastern part of Germany), response rate lower than $80 \%$; earlier studies (arbitrary stated cut-point 1995); proportion of young children (6-8 years versus 9-12 years); proportion of high parental education, and study design: whether the study was conducted within-towns, between-towns or a mixture.

All analyses were performed in STATA version 8.

\section{Results}

The distribution of parental education, confounders and potential sources of heterogeneity across countries are summarized in Table 1. The prevalence of higher education differed between countries, ranging from $13.7 \%$ in Germany to $67.1 \%$ in North America.

Final set of confounders

The final set of early life factors (Model II) included mother smoking during pregnancy (highest change of OR for low MMEF $-11.4 \%$ in the Netherlands and for low PEF $-13.8 \%$ in Poland), premature birth, low birth weight, attendance of kindergarten and breastfeeding. Other a priori confounders did not meet the set criteria and therefore they were not used for analysis. Controlling for these risk 
Table 1 Description of the studies and the study sample (in total 24,010 children)

\begin{tabular}{|c|c|c|c|c|c|c|c|c|c|}
\hline Country abbreviation $^{\mathrm{a}}$ & POL & HUN & SLO & CZE & NL & GER & A & US & All \\
\hline Number of study-areas & 4 & 5 & 4 & 4 & 24 & 3 & 8 & 24 & 76 \\
\hline Number of towns & 4 & 5 & 3 & 1 & 19 & 3 & 1 & 24 & 60 \\
\hline Year(s) of questionnaires and lung function & 96 & 96 & 96 & 96 & $97 / 98$ & $92 / 93$ & $96-98$ & $88-90$ & $88-98$ \\
\hline Number of children $(n)$ & 1,472 & 1,613 & 970 & 806 & 1,734 & 1,788 & 2,898 & 12,729 & 24,010 \\
\hline Male sex $(n)$ & 726 & 768 & 519 & 455 & 859 & 902 & 1,544 & 6,497 & 12,270 \\
\hline$\%$ from country sample & $(49.3)$ & $(47.6)$ & $(53.5)$ & $(56.5)$ & $(49.5)$ & $(50.4)$ & $(53.3)$ & $(51.0)$ & $(51.1)$ \\
\hline Age range selected for lung function testing & $9-12$ & $9-12$ & $9-12$ & $9-12$ & $7-12$ & $6-12$ & $6-11$ & $8-12$ & $6-12$ \\
\hline Age group 9-12 $(n)$ & 1,472 & 1,613 & 970 & 806 & 1,225 & 1,230 & 73 & 12,057 & 19,446 \\
\hline$\%$ from country sample & $(100.0)$ & $(100.0)$ & $(100.0)$ & $(100.0)$ & $(70.6)$ & $(68.80)$ & $(2.5)$ & $(94.7)$ & $(81.0)$ \\
\hline High parental education $(n)$ & 259 & 418 & 400 & 205 & 704 & 233 & 999 & 8,207 & 11,425 \\
\hline$\%$ from country sample & $(17.6)$ & $(25.9)$ & $(41.2)$ & $(25.4)$ & $(40.6)$ & $(13.0)$ & $(34.5)$ & $(64.5)$ & $(47.6)$ \\
\hline \multicolumn{10}{|l|}{ Early life factors (Model II) } \\
\hline Mother smoking during pregnancy $(n)$ & 371 & 201 & 63 & 72 & 464 & 86 & 511 & 3,296 & 5,064 \\
\hline$\%$ from country sample & $(25.2)$ & $(12.5)$ & $(6.5)$ & $(8.9)$ & $(26.8)$ & $(4.8)$ & $(17.6)$ & $(28.9)$ & $(21.1)$ \\
\hline Premature birth $(n)$ & 150 & 134 & 78 & 65 & 170 & 132 & - & 588 & 1,317 \\
\hline$\%$ from country sample & $(10.2)$ & $(8.3)$ & $(8.0)$ & $(8.1)$ & $(9.8)$ & (7.4) & - & $(4.6)$ & $(5.5)$ \\
\hline Low birth weight $<2.3 \mathrm{~kg}(n)$ & 162 & 145 & 85 & 67 & 189 & 149 & - & 591 & 1,388 \\
\hline$\%$ from country sample & $(11.0)$ & $(9.0)$ & $(8.8)$ & $(8.3)$ & $(10.9)$ & $(8.3)$ & - & $(4.6)$ & $(5.8)$ \\
\hline Breastfeeding $(n)$ & 997 & 1,196 & 860 & 691 & 1,098 & 1,387 & - & - & 6,229 \\
\hline$\%$ from country sample & $(67.7)$ & $(74.1)$ & $(88.7)$ & $(85.7)$ & $(63.3)$ & $(77.6)$ & - & - & $(25.9)$ \\
\hline Attendance kindergarten $(n)$ & 221 & 329 & 390 & 242 & 160 & 1,221 & - & - & 2,563 \\
\hline$\%$ from country sample & $(15.0)$ & $(20.4)$ & $(40.2)$ & $(30.0)$ & $(9.2)$ & $(68.3)$ & - & - & $(10.7)$ \\
\hline \multicolumn{10}{|l|}{ Other potential confounders (Model III) } \\
\hline Current smoker in a household $(n)$ & 983 & 913 & 521 & 471 & 982 & 823 & 1,690 & 6,282 & 12,665 \\
\hline$\%$ from country sample & $(66.8)$ & $(56.6)$ & $(53.7)$ & $(58.4)$ & $(56.6)$ & $(46.0)$ & $(58.3)$ & $(49.4)$ & $(52.7)$ \\
\hline Pet ownership ever $(n)$ & 939 & 749 & 441 & 477 & 1,301 & 1,161 & 1,317 & - & 6,385 \\
\hline$\%$ from country sample & $(63.8)$ & $(46.4)$ & $(45.5)$ & $(59.2)$ & $(75.0)$ & $(64.9)$ & $(45.4)$ & - & $(26.6)$ \\
\hline Crowding $(n)$ & 258 & 211 & 111 & 87 & 221 & 267 & - & 2,123 & 3,278 \\
\hline$\%$ from country sample & $(17.5)$ & $(13.1)$ & $(11.4)$ & $(10.8)$ & $(12.7)$ & $(14.9)$ & - & $(16.7)$ & $(13.7)$ \\
\hline Overweight and obesity $(n)$ & 273 & 254 & 149 & 186 & 291 & 289 & 558 & 4,219 & 6,219 \\
\hline$\%$ from country sample & $(18.6)$ & $(15.8)$ & $(15.4)$ & $(23.1)$ & $(16.8)$ & $(16.2)$ & $(19.3)$ & $(33.2)$ & $(25.9)$ \\
\hline \multicolumn{10}{|l|}{ Variables for exploring heterogeneity } \\
\hline East-West $($ East $=1$, West $=0)$ & 1 & 1 & 1 & 1 & 0 & 1 & 0 & 0 & \\
\hline Response rate $<0.8($ yes $=1)$ & 1 & 1 & 1 & 1 & 1 & 0 & 0 & 0 & \\
\hline Post 1995 study $($ yes $=1)$ & 1 & 1 & 1 & 1 & 1 & 0 & 1 & 0 & \\
\hline $\begin{array}{l}\text { Proportion of young children } \\
\qquad(<20 \%=1, \text { med }=2,>80 \%=3)\end{array}$ & 2 & 2 & 2 & 2 & 2 & 2 & 3 & 1 & \\
\hline Proportion high parental education $<30 \%$ (yes $=1$ ) & 0 & 0 & 1 & 0 & 1 & 0 & 1 & 1 & \\
\hline Study design within-towns, between-, mixture (W, B, M) & $\mathrm{B}$ & $\mathrm{B}$ & M & $\mathrm{W}$ & M & $\mathrm{B}$ & $\mathrm{W}$ & $\mathrm{B}$ & \\
\hline
\end{tabular}

${ }^{a}$ Country abbreviation: POL Poland (CESAR study)—Leonardi et al. [13]; HUN Hungary (CESAR study) —Leonardi et al. [13]; SLO Slovakia (CESAR study)—Leonardi et al. [13]; CZE Czech R (CESAR study)—Leonardi et al. [13]; NL 24 school study, Netherlands—Janssen et al. [16]; GER Bitterfeldt study, Germany-Heinrich et al. [15]; A Linz Study, Austria-Neuberger et al. [14]; US 24 city study, North AmericaRaizenne et al. [12]

factors changed odds ratios only slightly, e.g. LRI in first 2 years of life with the greatest change being $-6.6 \%$ for low MMEF in Poland. A still weaker impact was made by controlling for parental history of asthma.

The final set of other risk factors to be adjusted for in Model III were BMI (highest change of OR for low FEV1 by $15.7 \%$ in Poland), presence of a smoker in the household (highest change of OR for low FVC $-20.3 \%$ in the Czech Republic), pet ownership (changed OR for low PEF by $-14.8 \%$ in Hungary) and overcrowding (highest change of OR for low PEF $-20.0 \%$ in Poland). Presence of mould/ moisture in the child's home, use of gas for cooking or 
gas/kerosene/oil heater and infection prior to test modified the relationship between low parental education and health outcomes only little, so that were not included into the final set of confounders.

\section{Continuous lung function analyses}

The association between continuous lung function parameters and low parental education was not consistent across the countries (Fig. 1). The results of the fully adjusted linear regression model varied between countries, ranging for FEV1 and FVC from changes of $-1.09 \%$ and $-1.08 \%$ respectively in Germany to changes of $+1.43 \%$ and $+1.33 \%$ respectively in Poland; and MMEF changes ranged from $-1.66 \%$ in Slovakia to $+1.70 \%$ in Poland. The most consistent decrease was found for PEF, ranging from $-2.80 \%$ in the USA to $-1.14 \%$ in Hungary and the results were statistically significant in five out of eight countries.

In the basic model low parental education was associated with a significant overall mean decrease of PEF $(-2.2 \%)$ and
Fig. 1 Forest plots for the effect estimates of low parental education. Effect estimates (\% change) of low parental education ( $)$ basic model, early life factors adjusted model, $\Delta$ fully adjusted model). Vertical lines indicate null position. Horizontal lines represent $95 \%$ confidence intervals. Combined indicate random effect summary estimates (\% change) and $95 \%$ confidence interval
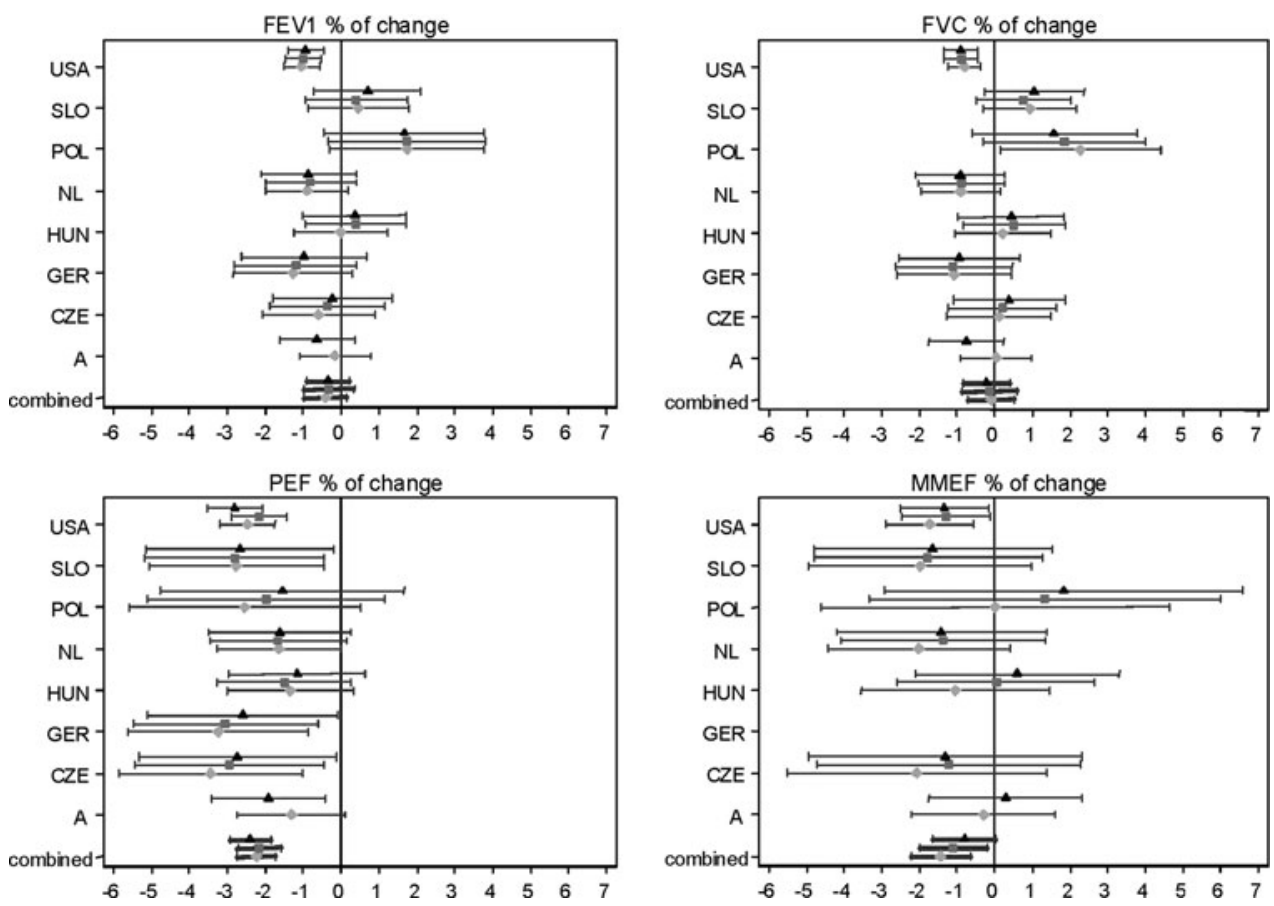

Table 2 The mean percentage change of lung function parameters in association with low parental education for each outcome from the basic (adjusted for age, sex, height, weight and technician), early life factors and all confounder adjusted models

\begin{tabular}{|c|c|c|c|}
\hline & $\begin{array}{l}\text { Model I } \\
\text { Basic model } \\
\text { Change \% }(95 \% \mathrm{CI})\end{array}$ & $\begin{array}{l}\text { Model II } \\
\text { Early life factors adjusted } \\
\text { Change \% }(95 \% \mathrm{CI})\end{array}$ & $\begin{array}{l}\text { Model III } \\
\text { Confounder adjusted } \\
\text { Change \% (95\% CI })\end{array}$ \\
\hline \multicolumn{4}{|l|}{ FEVI } \\
\hline Estimate (random) & $-0.4(-1.0 ; 0.1)$ & $-0.3(-1.0 ; 0.3)$ & $-0.4(-0.9 ; 0.2)$ \\
\hline \multicolumn{3}{|l|}{ 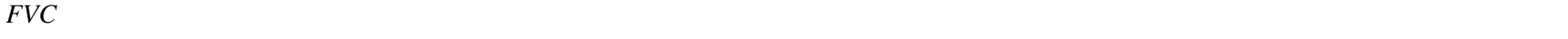 } & 0.112 \\
\hline Estimate (random) & $-0.1(-0.7 ; 0.5)$ & $-0.1(-0.9 ; 0.6)$ & $-0.2(-0.8 ; 0.4)$ \\
\hline p-Heterogeneity & 0.013 & 0.017 & 0.028 \\
\hline \multicolumn{4}{|l|}{$P E F$} \\
\hline Estimate (random) & $-2.2(-2.7 ;-1.7)$ & $-2.2(-2.7 ;-1.6)$ & $-2.4(-2.9 ;-1.8)$ \\
\hline p-Heterogeneity & 0.600 & 0.915 & 0.688 \\
\hline \multicolumn{4}{|l|}{$M M E F$} \\
\hline Estimate (random) & $-1.4(-2.2 ;-0.6)$ & $-1.1(-2.0 ;-0.2)$ & $-0.8(-1.6 ; 0.04)$ \\
\hline p-Heterogeneity & 0.862 & 0.835 & 0.604 \\
\hline
\end{tabular}

$P$-values for evidence of heterogeneity between study-specific results 
$\operatorname{MMEF}(-1.4 \%)$ with no indication of heterogeneity between studies (Table 2). No association with FVC and a borderline significant association with FEV1 were found in Model I with evidence of heterogeneity $(P<0.10)$ for both these outcomes-Table 2 . After controlling for early life factors the effect of low parental education on lung function parameters slightly decreased except for PEF. Further adjustment for all other potential risk factors confirmed the only significant association between low education and PEF $(-2.4 \%)$ and still borderline significance with MMEF $(-0.8 \%)$.

Low lung function analyses

The overall distribution of low lung function varied modestly between countries. The mean distribution of low FEV1 reached 6.5\%, low FVC 6.1\%, low PEF 5.2\% and low MMEF $10.6 \%$ across the countries (Table 3).

The mean odds ratios describing the association between parental education and prevalence of low lung function were statistically significant for FVC, PEF and MMEF and ranged from 1.18 for MMEF to 1.38 for PEF in the baseline Model I (Fig. 2). The associations with parental education persisted after additional adjustment for early life factors and other potential confounders, but were statistically significant only for PEF in Model III (Fig. 2). Mean odds ratios for FEV1 were not statistically significant, with considerable between-country heterogeneity. The fully adjusted model gave slightly lower mean odds ratios for FVC and MMEF compared with the basic model. For PEF, where the largest adverse effects were seen, the mean OR changed only slightly after adjustment from 1.38 (95\% CI
1.08-1.76), to 1.34 (95\% CI 1.06-1.70). The results indicate that the association with parental education could not be fully explained by the additional studied risk factors.

Analysis of heterogeneity of results

Country-specific results tended to be heterogeneous. We explored this heterogeneity, focussing on the low lung function measures. The significant source of heterogeneity of the results was the differences between the Western and Eastern countries for FEV1, and differences between countries with higher (more than $80 \%$ ) and lower responserate for PEF (Table 4).

The association between low FEV1 and low parental education appears to be confined to Western countries $(\mathrm{OR}=1.28 ; 95 \%$ CI $1.10-1.49)$, where the ORs varied from 1.20 (95\% CI 1.01-1.43) in North America to 1.56 (95\% CI 0.96-2.53) in the Netherlands (Table 4, Fig. 2).

The same East-West difference was not apparent for low FVC, MMEF or PEF.

All other study characteristics (proportion of young children, proportion of high parental education, and study design) could not explain heterogeneity between countryspecific estimates.

\section{Sensitivity analysis}

The impact of missing confounding variables in some studies on the meta-analysis results was explored in sensitivity analyses. Study results for each of the health outcome were compared between groups of studies with the

Table 3 Description of distribution of low lung function accross the countries

\begin{tabular}{|c|c|c|c|c|c|c|c|c|c|}
\hline Abbreviation & POL & HUN & SLO & CZE & NL & GER & A & US & All \\
\hline Low FEV1 ${ }^{\text {a }}(N)$ & 42 & 66 & 50 & 30 & 102 & 103 & 152 & 802 & 1,347 \\
\hline$\%$ of study sample & 6.8 & 5.3 & 5.2 & 3.7 & 6.3 & 6.5 & 6.4 & 7.0 & 6.5 \\
\hline Low FEV1 in high parental education (\%) & 11.0 & 5.9 & 6.0 & 2.9 & 5.0 & 4.6 & 4.9 & 6.7 & 6.3 \\
\hline Low FEV in low parental education $(\%)$ & 5.8 & 5.1 & 4.6 & 4.0 & 7.3 & 6.8 & 7.3 & 7.8 & 6.8 \\
\hline Low FVC $^{\text {a }}(N)$ & 38 & 77 & 46 & 29 & 101 & 150 & 140 & 681 & 1,262 \\
\hline$\%$ of study sample & 6.4 & 6.4 & 4.7 & 3.6 & 6.2 & 8.6 & 5.9 & 6.0 & 6.1 \\
\hline Low FVC in high parental education (\%) & 7.2 & 6.3 & 5.0 & 4.4 & 4.7 & 4.8 & 5.0 & 5.4 & 5.3 \\
\hline Low FVC in low parental education $(\%)$ & 6.2 & 6.4 & 4.6 & 3.3 & 7.4 & 9.3 & 6.4 & 7.2 & 6.9 \\
\hline Low $\operatorname{PEF}^{\mathrm{a}}(N)$ & 20 & 28 & 59 & 32 & 75 & 74 & 127 & 669 & 1,084 \\
\hline$\%$ of study sample & 3.4 & 2.3 & 6.1 & 4.0 & 4.6 & 4.3 & 4.0 & 5.9 & 5.2 \\
\hline Low PEF in high parental education $(\%)$ & 1.7 & 2.7 & 6.5 & 2.9 & 4.4 & 1.7 & 3.8 & 4.8 & 4.6 \\
\hline Low PEF in low parental education $(\%)$ & 3.8 & 2.2 & 5.8 & 4.3 & 4.8 & 4.7 & 6.3 & 8.1 & 5.9 \\
\hline Low $\operatorname{MMEF}^{\mathrm{a}}(N)$ & 60 & 104 & 92 & 81 & 166 & - & 276 & 1,240 & 2,019 \\
\hline$\%$ of study sample & 10.1 & 8.6 & 9.5 & 10.0 & 10.2 & - & 11.6 & 10.9 & 10.6 \\
\hline Low MMEF in high parental education (\%) & 11.7 & 6.0 & 9.0 & 9.3 & 8.8 & - & 10.4 & 10.4 & 10.1 \\
\hline Low MMEF in low parental education (\%) & 9.8 & 9.6 & 9.8 & 10.3 & 11.3 & - & 12.3 & 11.9 & 11.3 \\
\hline
\end{tabular}

${ }^{\text {a }}$ Low lung function is measured lung function less than $85 \%$ (FVC, FEV1) or 75\% (PEF, MMEF) of predicted lung function based upon age, sex, height and weight 
Fig. 2 Forest plots for the association between low FEV1, FVC, PEF and MMEF and low parental education. Effect estimates (Odds ratios) of low parental education ( $)$ basic model, early life factors adjusted model, $\Delta$ fully adjusted model). Vertical lines indicate null position $(\mathrm{OR}=1)$. Horizontal lines represent $95 \%$ confidence intervals. Combined indicate random effect summary estimates (OR) and 95\% confidence interval
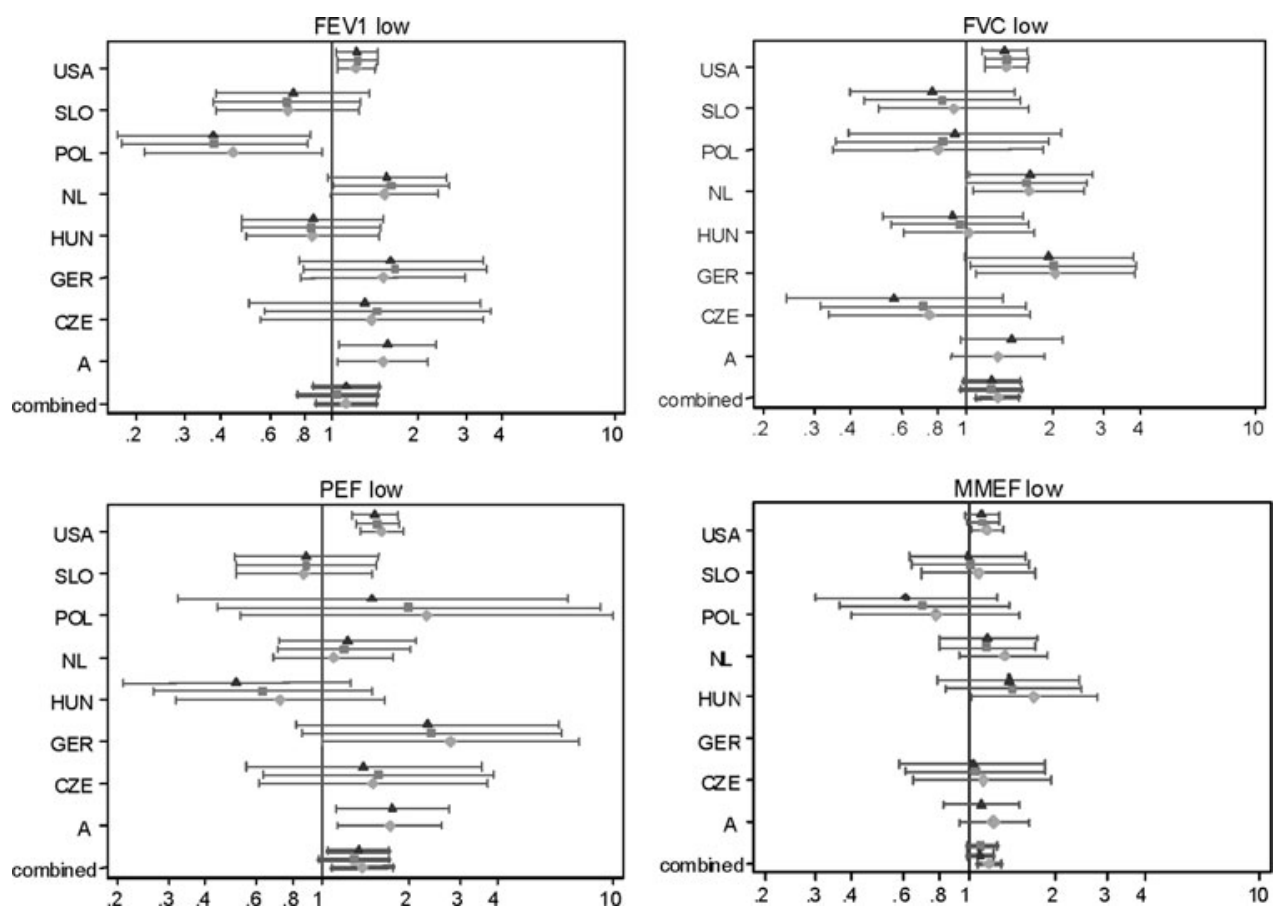

Table 4 Sources of heterogeneity of results - comparison of mean odds ratios for the association between low lung function and low parental education across subgroups of countries

\begin{tabular}{llll}
\hline & $\begin{array}{l}\text { No. of } \\
\text { studies }\end{array}$ & $\begin{array}{l}\text { Mean odds } \\
\text { ratio }(95 \% \mathrm{CI})\end{array}$ & $P$-value \\
\hline $\begin{array}{l}\text { FEV1 low } \\
\text { East }^{\mathrm{a}}\end{array}$ & 5 & $0.86(0.56-1.31)$ & \\
West & 3 & $\mathbf{1 . 2 8}(\mathbf{1 . 1 0}-\mathbf{1 . 4 9})$ & 0.040 \\
PEF low & & & \\
Response rate $\geq 80 \%{ }^{\mathrm{b}}$ & 3 & $\mathbf{1 . 5 6}(\mathbf{1 . 3 2}-\mathbf{1 . 8 4})$ & \\
Response rate $<80 \%$ & 5 & $1.01(0.73-1.40)$ & 0.020 \\
\hline
\end{tabular}

Only factors significantly associated with the size of the effect estimate are presented

${ }^{a}$ East Eastern countries-Poland, Hungary, Slovak Republic, Czech Republic, Germany; West Western countries-Netherlands, Austria, US

${ }^{\mathrm{b}}$ Response rate $<80 \%$-Poland, Hungary, Slovak Republic, Czech Republic, Netherlands; response rate $\geq 80 \%$-Germany, Austria, US

full availability of covariates, studies with five items missing (early life factors and overcrowding in Austria) and three items missing (breastfeeding, attendance of kindergarten and presence of pets in a household in North America). The sensitivity analyses indicated that the missing confounders in some studies did not significantly change the results.

Excluding the US data lead to broader confidence intervals, decreased \% change of lung function parameters (FEV1, FVC, MMEF) and loss of heterogeneity of results for FEV1. Indication of heterogeneity of results in the restricted data set has still been confirmed for FVC. Other results were found out regarding $\mathrm{PEF}$ - the point estimates for the percentage difference in fully adjusted models decreased from $-2.4 \%$ in the full dataset to $-1.9 \%$ in the restricted data set and heterogeneity decreased practically to zero. All the models for PEF (full data set, restricted data set and US data set itself) provided statistically significant results with narrow confidence intervals.

\section{Discussion}

This study found positive associations between low lung function and low parental education. Significant risk increases of low lung function parameters $(\mathrm{PEF} O \mathrm{OR}=1.38$, FVC OR $=1.28$, and MMEF OR $=1.18$ ) were associated with low parental education before inclusion of potential risk factors. Odds ratios for low FVC and MMEF were reduced and became statistically non-significant after adjustment for other risk factors, but the risk of low PEF remained persistent after adjustment, decreasing only slightly to $\mathrm{OR}=1.34$. The overall effect of SES over LF parameters was found. That only PEF remained significant is mostly due to the fact that PEF shows the smallest chance variation. So here the power to detect small effects is greatest.

Our study results support findings from other studies on socio-economic differences of lung function [5, 9, 10] mostly being carried out in the Western countries and showed that the social gradient is less pronounced in Eastern European countries.

In a previous analysis of the PATY data [17], adverse effects of low parental education were observed on 
prevalences of wheeze and nocturnal dry cough. The association with wheeze was mainly restricted to children from Eastern Europe, and the authors commented that different distributions of atopy and different patterns of link between wheeze, atopy, and SES in Eastern and Western countries might have played a role [17]. The EastWest effect modification for lung function, reported in the current paper, is in the opposite direction, with higher effects of low education occurring in the Western countries. We speculate that this may be partly due to a more pronounced link in Western countries between individuallevel socioeconomic status and area-level deprivation, with the latter-acting perhaps via factors such as housing quality, and ambient air pollution-having its own adverse effects on lung function [23].

Explanation of associations with education by other risk factors

The study results might be conservative. Inclusion of other risk factors may result in over-adjustment as part of the behaviours, e.g. smoking, or living conditions, e.g. mould in the home, may be in the causal chain from socio-economic conditions and health.

Low respiratory tract infection in early childhood had been hypothesized to be one of the risk factors [11], but in our multi-country study controlling for this risk factor changed odds ratios only slightly. The impact of early life factors on lung function data in our study confirmed the results of previous studies-maternal smoking during pregnancy [20], day-care attendance [24], and low-birth weight and premature birth [11].

Passive smoking is one of the most important confirmed risk factor of impaired lung function [20, 25, 26]. This was again confirmed in this study.

Brunekreef et al. [27] reported dampness and moulds to be a strong predictor of impaired lung function. But in our study mould/moisture did not relevantly affect the relationship between education and health outcomes.

Some studies reported SES differences in outdoor/ indoor air pollution [28-31]. However adverse effects on lung function are reversible with the improvement of air quality $[14,32]$. In spite of these reported class-specific differences of indoor exposure, the impact of gas use for cooking or use of unvented gas/kerosene/oil (water) heater had only a weak impact on relationships being investigated in our study.

Some studies reported racial differences to be a cause of different lung function outcomes [33, 34]. The information on race of children was not available in most of component studies. For the Austrian, the Netherlands and the US datasets the information on nationality/race was available, but it concerned only minorities representing around 3.5\% of the study sample in the US and the Netherlands. In the Austrian data nationality was based on "child's language" and minorities varied from 2.3 to $8.4 \%$. To avoid confounding of effect estimates caused by genetic aspects relating with the different race (in the US data) and ethnic differences probably interacting both with SES and culturally determined life-style only data on major population was analysed.

No comparative data on physical activity was available. Instead BMI was included into a set of potential confounders as the association between lung function parameters and overweight in children was confirmed by several studies [35-37].

We have to acknowledge that our variable 'low education' may not be fully equivalent across all countries, and that quantitative results from pooling country-specific effect estimates may be seen as indicative rather than as numeric absolutes. Gehring et al. reporting on parental education and respiratory symptoms in the PATY study [17], expressed a confidence that parental education really reflected the children's SES, based on consistency of the association between parental education and crowding. Furthermore, our analyses of heterogeneity did not find differences of results between countries with different proportions of high parental education. However, the country-specific effect estimates themselves and the patterns of association seen across them, remain important results, besides the mean estimates presented.

It is worthy to notice that education as previously defined [17] is a proxi of SES for a broader range of possible risk factors for low lung function that can be modified, including quality of housing, poor sanitation, air pollution in a living area, ETS exposure, diet, physical activities, and access to health care. Even when a range of these risk factors that are linked to SES (mother smoking in pregnancy, premature birth/low birth weight, breastfeeding, attending kindergarten, presence of current smoker in a household, pet ownership ever and overcrowding) are included in the model there remains an effect of low parental education that is not explained by these specific risk factors. That proxy SES measures, such as low education, can explain more variation in ill-health than the sum of the known individual risk factors, illustrates precisely the strength of SES as a concept of health relevance.

\section{East-West differences}

Analysis of heterogeneity confirmed East-West differences in study results for low FEV1 and low FVC and differences between groups of countries according the response-rate (below/above 80\%) for low PEF. Considering that higher response rates were seen in the US, Austria, and German studies, and East-West and response-rate differences greatly 
overlapped, it can be assumed that the differences are caused more likely by East-West than response-rate differences.

East-West differences were also found in analyses of wheeze and low parental education in the earlier paper [17]. The opposite East-West pattern was seen in the present analysis - higher risks of low FEV1 and low FVC were seen in children from less educated families in the Western countries. The observed East-West differences in relationship between low education and lung function might be ascribed to differences in factual status [38, 39] regarding occupational class, material resources, life-style and behavior patterns, with education in Eastern countries not necessarily a good indicator of affluence due to low consistency of SES. This speculation is supported by Davey-Smith et al. [40] who argued that material resources related to occupational social class rather than culturally based sources related to education should be key determinants of socio-economic differentials. However, information on occupational social class of parents was not available for most studies.

Although the data were acquired in the period from 1988 to 1998 , the authors are confident of its relevance. Such large datasets, especially for the US population have not been collected since that period. The East-West differences in social health inequalities might have changed since that time. Education is having a much more important role in SES in the Eastern countries. But the argument of material conditions as key determinant of socio-economic health differences remains, and this still persists between Western and Eastern countries.

\section{Conclusions}

The results of the study confirmed a higher risk of low lung function in children from less educated families. The associations with parental education were more pronounced in the Western countries, while in the Eastern countries the direction of the association was not consistent, which might be explained by the low consistency of SES in the former socialist countries. Although social gradients were attenuated after adjusting for several known risk factors, these risk factors could not completely explain the social gradient in lung function.

Acknowledgments The PATY study was funded by the EU 5th Framework Quality of Life Programme, Contract No. QLRT-200102544. The authors would like to thank the scientists and technical staff involved in the original studies.

Open Access This article is distributed under the terms of the Creative Commons Attribution Noncommercial License which permits any noncommercial use, distribution, and reproduction in any medium, provided the original author(s) and source are credited.

\section{References}

1. Marmot MG, Shipley MJ, Rose G. Inequalities in death-specific explanations of a general pattern? Lancet. 1984;1:1003-6.

2. Strand BH, Tverdal A. Can cardiovascular risk factors and lifestyle explain the educational inequalities in mortality from ischaemic heart disease and from other heart diseases? 26 year follow up of 50, 000 Norwegian men and women. J Epidemiol Community Health. 2004;58:705-9.

3. Van Lenthe FJ, Borrell LN, Costa G, et al. Neighbourhood unemployment and all cause mortality: a comparison of six countries. J Epidemiol Community Health. 2005;59:231-7.

4. Bakke PS, Hanoa R, Gulsvik A. Educational level and obstructive lung disease given smoking habits and occupational airborne exposure: a Norwegian community study. Am J Epidemiol. 1995;141:1080-8.

5. Prescott E, Lange P, Vestbo J. Socioeconomic status, lung function and admission to hospital for COPD: results from the Copenhagen City Heart Study. Eur Respir J. 1999;13:1109-14.

6. Hegewald MJ, Crapo RO. Socioeconomic status and lung function. Chest. 2007;132:1608-14.

7. Jackson B, Kubzansky LD, Cohen S, et al. CARDIA Study. A matter of life and breath: childhood socioeconomic status is related to young adult pulmonary function in the CARDIA study. Int J Epidemiol. 2004;33:271-8.

8. Lawlor DA, Ebrahim S, Smith GD. Association between selfreported childhood socioeconomic position and adult lung function: findings from the British Women's Heart and Health Study. Thorax. 2004;59:199-203.

9. Vedal S, Schenker MB, Samet JM, et al. Risk factors for childhood respiratory disease. Analysis of pulmonary function. Am Rev Respir Dis. 1984;130:187-92.

10. Demissie K, Ernst P, Hanley JA, et al. Socioeconomic status and lung function among primary school children in Canada. Am J Respir Crit Care Med. 1996;153:719-23.

11. Lercher P, Schmitzberger R. Birth weight, education, environment, and lung function at school age: a community study in an alpine area. Eur Respir J. 1997;10:2502-7.

12. Raizenne M, Neas LM, Damokosh AI, et al. Health effects of acid aerosols on North American children: pulmonary function. Environ Health Perspect. 1996;104:506-14.

13. Leonardi GS, Houthuijs D, Nikiforov B, et al. Respiratory symptoms, bronchitis and asthma in children of Central and Eastern Europe. Eur Respir J. 2002;20:890-8.

14. Neuberger M, Moshammer H, Kundi M. Declining ambient air pollution and lung function improvement in Austrian children. Atmos Environ. 2002;36:1733-6.

15. Heinrich J, Hoelscher B, Wjst M, et al. Respiratory diseases and allergies in two polluted areas in East Germany. Environ Health Perspect. 1999;107:53-62.

16. Janssen NA, Brunekreef B, van Vliet $P$, et al. The relationship between air pollution from heavy traffic and allergic sensitization, bronchial hyperresponsiveness, and respiratory symptoms in Dutch schoolchildren. Environ Health Perspect. 2003;111: 1512-8.

17. Gehring U, Pattenden S, Slachtova H, et al. Parental education and children's respiratory and allergic symptoms in the Pollution and the Young (PATY) study. Eur Respir J. 2006;27:95-107.

18. American Thoracic Society. Standardization of spirometry1987 update. Am Rev Respir Dis. 1987;136:1285-98.

19. Standardized lung function testing. Official statement of the European Respiratory Society. Eur Respir J Suppl. 1993;16:1-100.

20. Moshammer H, Hoek G, Luttmann-Gibson H, et al. Parental smoking and lung function in children: an international study. Am J Respir Crit Care Med. 2006;173:1255-63. 
21. Vidmar S, Carlin J, Hesketh K, Cole T. Standardizing anthropometric measures in children and adolescents with new functions for egen. Stata J. 2004;4:50-5.

22. DerSimonian R, Laird N. Meta-analysis in clinical trials. Control Clin Trials. 1986;7:177-88.

23. Shohaimi S, Welch A, Bingham S, Luben R, Day N, Wareham N, Khaw KT. Area deprivation predicts lung function independently of education and social class. Eur Respir J. 2004;24: 157-61.

24. Demissie K, Ernst P, Joseph L, et al. The role of domestic factors and day-care attendance on lung function of primary school children. Respir Med. 1998;92:928-35.

25. Forastiere F, Agabiti N, Corbo GM, et al. Passive smoking as a determinant of bronchial responsiveness in children. Am J Respir Crit Care Med. 1994;149:365-70.

26. Ware JH, Dockery DW, Spiro A 3rd, et al. Passive smoking, gas cooking, and respiratory health of children living in six cities. Am Rev Respir Dis. 1984;129:366-74.

27. Brunekreef B, Dockery DW, Speizer FE, et al. Home dampness and respiratory morbidity in children. Am Rev Respir Dis. 1989; 140:1363-7.

28. Heinrich J, Mielck A, Schäfer I, et al. Social inequality and environmentally-related diseases in Germany: review of empirical results. Soz Praventivmed. 2000;45:106-18.

29. Eroshina K, Danishevski K, Wilkinson P, et al. Environmental and social factors as determinants of respiratory dysfunction in junior schoolchildren in Moscow. J Public Health. 2004;26: 197-204.

30. Prescott E, Vestbo J. Socioeconomic status and chronic obstructive pulmonary disease. Thorax. 1999;54:737-41.

31. Zeka A, Melly SJ, Schwartz J. The effects of socioeconomic status and indices of physical environment on reduced birth weight and preterm births in Eastern Massachusetts. Environ Health. 2008;7:60.
32. Frye C, Hoelscher B, Cyrys J, et al. Association of lung function with declining ambient air pollution. Environ Health Perspect. 2003;111:383-7.

33. Cunningham J, Dockery DW, Gold DR, et al. Racial differences in the association between maternal smoking during pregnancy and lung function in children. Am J Respir Crit Care Med. 1995;152:565-9.

34. Harik-Khan RI, Muller DC, Wise RA. Racial difference in lung function in African-American and White children: effect of anthropometric, socioeconomic, nutritional, and environmental factors. Am J Epidemiol. 2004; 160:893-900.

35. Chu YT, Chen WY, Wang TN, Tseng HI, Wu JR, Ko YC. Extreme BMI predicts higher asthma prevalence and is associated with lung function impairment in school-aged children. Pediatr Pulmonol. 2009;44:472-9.

36. Spathopoulos D, Paraskakis E, Trypsianis G, Tsalkidis A, Arvanitidou V, Emporiadou M, et al. The effect of obesity on pulmonary lung function of school aged children in Greece. Pediatr Pulmonol. 2009;44:273-80.

37. Lazarus R, Colditz G, Berkey CS, Speizer FE. Effects of body fat on ventilatory function in children and adolescents: cross-sectional findings from a random population sample of school children. Pediatr Pulmonol. 1997;24(3):187-94.

38. Hauser RM, Warren JR. Socioeconomic indexes for occupations: a review, update, and critique. Sociol Methodol. 1997;27:177-298.

39. Ganzeboom HBG, Treiman DJ. Three internationally standardised measures for comparative research on occupational status. In: Hoffmeyer-Zlotnik JHP, Wolf Ch, editors. Advances in crossnational comparison. A European working book for demographic and socio-economic variables. New York: Kluwer/Plenum; 2003. p. 159-94.

40. Davey-Smith G, Hart C, Hole D, et al. Education and occupational social class: which is the more important indicator of mortality risk? J Epidemiol Community Health. 1998;52:153-60. 